\title{
RECONHECIMENTO ETNOECOLÓGICO SOBRE O TUBARÃO-MANGONA CARCHARIAS TAURUS SOB A PERSPECTIVA DE PESCADORES ARTESANAIS DA GRANDE FLORIANÓPOLIS-SC, BRASIL
}

\author{
ETHNOECOLOGICAL RECOGNITION OF THE SAND TIGER SHARK CARCHARIAS TAURUS FROM THE \\ PERSPECTIVE OF ARTISANAL FISHERMEN FROM THE BIG FLORIANÓPOLIS-SC, BRAZIL
}

\section{RECONOCIMIENTO ETNOECOLOGICO SOBRE EL TIBURÓN TORO BACOTA CARCHARIAS TAURUS DESDE LA PERSPECTIVA DE PESCADORES ARTESANALES DEL GRAN FLORIANÓPOLIS, BRASIL}

Monique Cardoso Santos Edson Faria-Junior ${ }^{2}$

\section{Resumo}

Renato Hajenius Aché de Freitas

Estudos com populações tradicionais pesqueiras são importantes fontes de resgate de conhecimento sobre espécies ameaçadas. O tubarão-mangona, Carcharias taurus, está criticamente em perigo (CR) no Brasil. Assim, buscando elucidar aspectos etnoecológicos sobre a espécie e verificar possíveis alterações nas suas populações na Grande Florianópolis, 24 pescadores artesanais, especialistas na pesca da espécie, foram entrevistados. Demonstramos que a espécie foi amplamente capturada no passado, com evidente declínio das capturas em um curto espaço temporal, demonstrando a suscetibilidade desta espécie frente à sobrepesca. Ressalta-se também que os entrevistados não mais a capturam atualmente. A pesca de $C$. taurus ocorria em regiões próximas a Ilha de Santa Catarina, corroborando com seus hábitos de vida costeiros. Sua dieta foi descrita como generalista, por consumirem diversas espécies de peixes, raias e lulas. A época de pesca ocorria nos meses de novembro a fevereiro, quando capturavam fêmeas grávidas, coincidindo com a literatura como possível período de reprodução. Contudo, apesar de quase a totalidade dos pescadores reconhecerem o declínio das populações de tubarão-mangona, somente $55 \%$ deles acredita que isso pode gerar problemas ambientais, demonstrando desconhecimento do papel ecológico de um predador de topo de cadeia no ambiente ou baixa intenção na conservação dessa espécie.

Palavras-chave: pesca artesanal; elasmobrânquio; conhecimento ecológico local; extinção local; conservação.

1 Laboratório de Biologia de Teleósteos e Elasmobrânquios (LABITEL), Departamento de Ecologia e Zoologia, Centro de Ciências Biológicas, Universidade Federal de Santa Catarina, Florianópolis, Brasil. https://orcid.org/oooo-0oo1-7452-4227 e https://orcid.org/oooo-0002-9053-9186

2 Laboratório de Ecologia de Ambientes Recifais (LABAR), Departamento de Ecologia e Zoologia, Centro de Ciências Biológicas, Universidade Federal de Santa Catarina, Florianópolis, Brasil. https:// orcid.org/oooo-0001-9133-8303 


\begin{abstract}
Studies with traditional fishing populations are important sources to access the knowledge about threatened species. The sand tiger shark, Carcharias taurus, is critically endangered (CR) in Brazil. Thus, searching to elucidate ethnoecological aspects about the species and to verify possible alterations in their populations in Florianópolis and surroundings, 24 artisanal fishers, specializing to fish this species, were interviewed. We demonstrate that the species has been widely fish in the past, with a clear decline in catches in a short time, demonstrating the susceptibility of this species to overfishing. It is also noteworthy that respondents no longer capture it today nowadays. The fishing of $C$. taurus occurred in regions near to Santa Catarina Island, corroborating with its coastal life habits. It diet was described as generalist, because it consumes several species of fish, rays and squid. The fishing season occurred from November to February, when they captured pregnant females, coinciding with the literature as a possible reproduction period. However, although almost all of the fishermen recognize the decline of mangona shark's populations, only $55 \%$ of them believes it can lead to environmental problems, showing a lack of awareness of the ecological role of a top-predator in the environment or low intention to conserve this species.
\end{abstract}

Keywords: artisanal fishing; elasmobranch; local ecological knowledge; local extinction; conservation.

\title{
Resumen
}

Estudios con poblaciones tradicionales pesqueras son importantes fuentes de rescate de conocimiento sobre especies amenazadas. El tiburón toro bacota, Carcharias taurus, está críticamente en peligro (CR) en Brasil. De esta forma, buscando aclarar aspectos etnoecologicos sobre la especie y verificar posibles alteraciones en sus poblaciones en la Gran Florianópolis, 24 pescadores artesanales, especialistas en pesca de la especie, fueron entrevistados. Demostramos que la especie fue ampliamente capturada en el pasado, con evidente disminución de las capturas en un corto espacio temporal, demostrando la susceptibilidad de esta especie frente a la sobrepesca. Se resalta que los entrevistados no lo capturan más actualmente. La pesca del C. taurus ocurría en regiones próximas a la Isla de Santa Catarina, coincidiendo con sus hábitos de visa costeros. Su dieta fue descripta como generalista porque consumen diversas especies de peces, rayas y calamares. La época de pesca ocurría en los meses de noviembre a febrero, cuando capturaban hembras gestantes, coincidiendo con la literatura como posible período de reproducción. Sin embargo, a pesar de que casi la totalidad de los pescadores reconocen la disminución de poblaciones de tiburón mangona, solamente el $55 \%$ de ellos cree que eso puede generar problemas ambientales, demostrando desconocimiento del papel ecológico de un predador de la cima de la cadena ambiental o poca intención de conservación de esa especie.

Palabras llaves: pesca artesanal; elasmobranquio; conocimiento ecológico local; extinción local; conservación. 


\section{INTRODUÇÃO}

As capturas de tubarões giram em torno de 63 a 273 milhões de indivíduos por ano em uma escala global (Barbosa-Filho et al. 2014). O potencial pesqueiro do Brasil vem tomando lugar de destaque, quando em uma curta janela de tempo os elasmobrânquios deixaram de ser capturados majoritariamente como fauna acompanhante (by-catch), passando a ser alvo de pesca, e crescendo em número nos desembarques (Lessa et al. 2005). Segundo a FAO (Food and Agriculture Organization oft he United Nations ), as importações desses animais no Brasil chegaram a $21 \mathrm{mil}$ toneladas anuais na última década (Dente e Clarke 2015). A partir dos anos 2000 esses valores justificaram ao país a categoria de "Major Shark Fishing State", se tornando um dos seis países que mais capturam elasmobrânquios no mundo (Lack e Sant 2006). E não somente a pesca em escala industrial é importante, uma vez que a pesca artesanal contribui com $45 \%$ do total de pescados produzidos no país (Bornatowski et al. 2018).

Em Santa Catarina, a pesca artesanal é uma importante fonte de renda e subsistência para as populações tradicionais litorâneas (Bannwart 2014). Os elasmobrânquios representavam cerca de 12,7\% das capturas da pesca artesanal até a década de 90 (Costa e Chaves 2006). Esta modalidade de pesca é realizada pelas populações tradicionais, as quais são conhecidas por utilizar mão de obra familiar, ocupar o espaço e utilizar os recursos naturais principalmente para fins de subsistência (Arruda 1999). A sua inserção social, cultural, religiosa e econômica é proveniente de suas relações íntimas com a natureza (Diegues et al. 200o; Drew 2005; Pereirae Diegues 2010). No decorrer dessas relações, os saberes sobre o ecossistema e sobre os recursos ao qual fazem uso são adquiridos e transmitidos de forma empírica, podendo ser denominados como conhecimento ecológico tradicional (TEK - Traditional Ecological Knowledge) (Johnson 1992; Drew 2005). Quando na ausência de dados pretéritos sobre esforço pesqueiro de determinadas espécies, os pescadores são, muitas vezes, as únicas fontes para entendermos as mudanças que ocorreram no meio ambiente ao longo dos anos (Johannes et al. 2008). Desta forma, estudos etnoecológicos, e que utilizam uma abordagem cronológica sobre a história da pesca de determinados grupos, constitui importante ferramenta para a identificação de fatores responsáveis pelo declínio dos mesmos (Reis-Filho et al. 2016).

Entre as espécies de elasmobrânquios que possuem algum registro histórico em Santa Catarina sobre as suas populações, está o tubarão-mangona, Carcharias taurus. Análises de composição faunística de dentes de tubarões em um sítio arqueológico, datado de aproximadamente 1000 anos antes do presente na cidade de Florianópolis, mostraram uma composição de $48 \%$ de dentes de $C$. taurus (Hayata et al. 2018). Na região da Grande Florianópolis esses animais sofreram com uma intensa pescaria por volta de 1980, através da pesca com rede de emalhe (IBAMA 2011). A pesca subaquática da espécie também foi expressiva na região desde a década de 60 . 
Por exemplo, foram registrados 118 ton para 1961 e 130 ton para 1981 de capturas de C. taurus de acordo com a Federação Catarinense de Pesca Submarina (Souza 1994).

Por se tratar de uma espécie com hábitos costeiros, esses tubarões são facilmente capturados pela pesca artesanal (Bornatowski et al. 2018). Somando a isso, as características biológicas como baixíssima fecundidade ( 1 a 2 filhotes a cada dois anos), maturação sexual tardia, ocorrendo de 10 a 12 anos para fêmeas e de 6 a 8 anos para machos, e padrão de crescimento lento, evidenciam grande suscetibilidade à sobre-exploração pesqueira (Compagno 1984; Branstetter e Musick 1994; Goldman et al. 2006). Consequentemente, esta espécie aparece na lista vermelha de animais ameaçados de extinção da IUCN (International Union for Conservation of Nature) (Pollard e Smith 2009), na categoria de vulnerável (VU). Aparece também na Portaria no 445, de 17 de dezembro de 2014 na categoria de Criticamente Ameaçada (CR), (ICMBio 2014). Assim, a sobrepesca, nas suas diferentes práticas, demonstra-se como o principal fator para o decréscimo das populações dessa espécie, como observado em outras espécies (Reis-Filho et al. 2016). Em decorrência desses fatores, objetivou-se uma investigação etnoecológica para resgatar os conhecimentos produzidos ao longo da história pesqueira de $C$. taurus, permitindo a inferência de possíveis causas do desaparecimento local da espécie em um curto espaço temporal em Santa Catarina. Tal investigação está atrelada a percepção ecológica das populações tradicionais de pesca em relação à conservação desta espécie e, consequentemente, de predadores de topo de cadeia alimentar em qualquer região.

\section{MATERIAL E MÉTODOS}

Área de Estudo- Comunidades de pescadores distribuídas nas praias da Ilha de Santa Catarina: Ponta das Canas, Ingleses, Barra da Lagoa, Campeche, Armação do Pântano do Sul, Pântano do Sul e no litoral do Município de Governador Celso Ramos.

Coleta de Dados- Entre os anos de 2016 e 2017, 24 pescadores artesanais foram entrevistados, somente após terem afirmado conhecer ou ter pescado o tubarãomangona, ou serem indicados como pescadores de tubarão-mangona por outros membros da comunidade, formando assim uma rede de especialistas (Baldin e Munhoz 2011). Para confirmação, os entrevistados precisaram reconhecer a espéciefoco do estudo em um catálogo de imagens com 48 espécies de tubarões presentes, onde apenas os nomes científicos estavam expostos. A Figura 1 mostra a lâmina desse catálogo que continha a espécie-foco. 

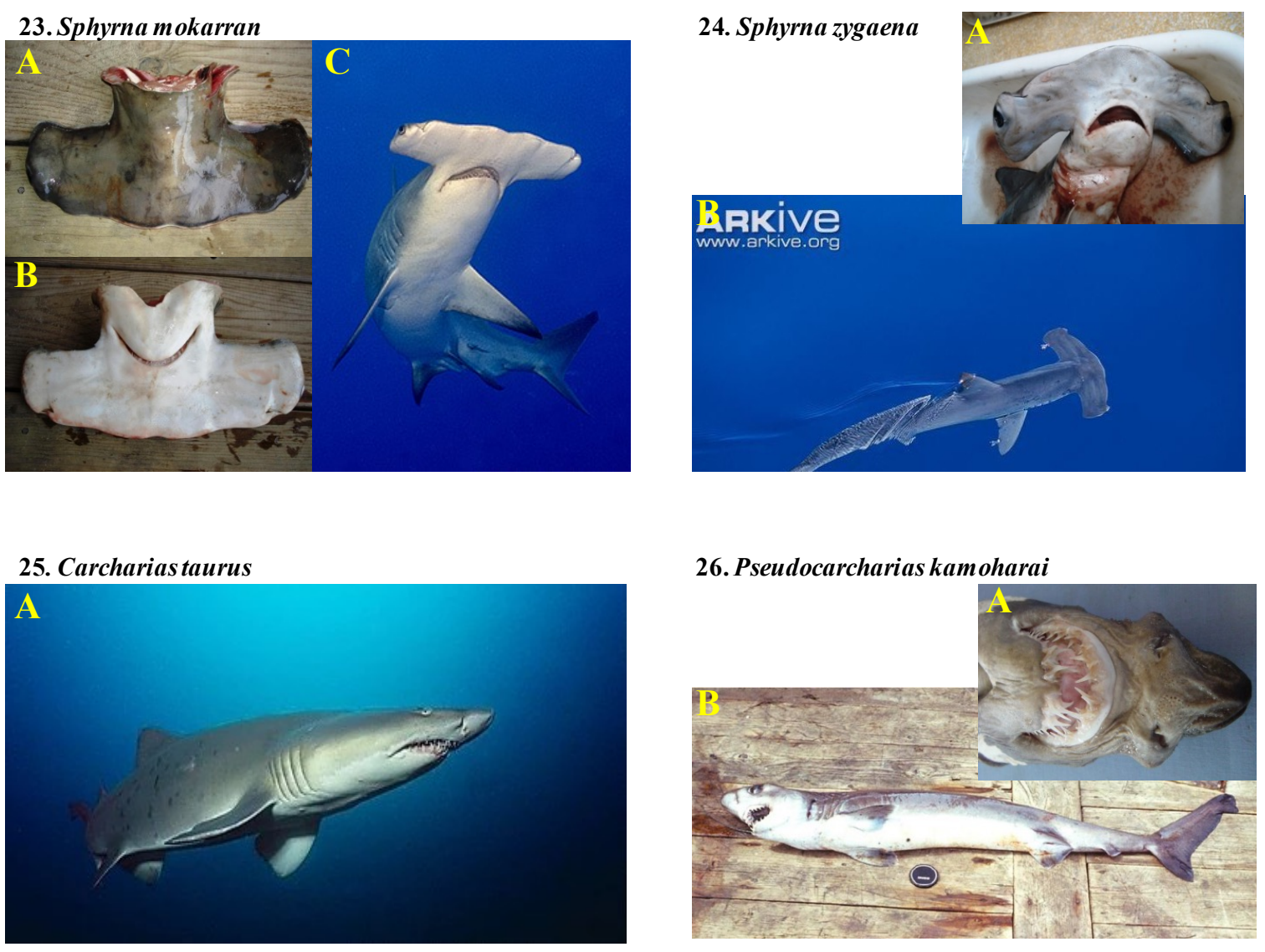

Figura 1. Lâmina de um catálogo de 48 fotos de tubarões com a espécie Carcharias taurus. Imagens retiradas de diferentes sites. Autorias: 23A e 23B de Trevor Meyer, $23 \mathrm{C}$ de Karl Dietz, 24A de Hugo Bornatowski, 24B de François Gohier, 25A de Howard Hall, 26A de Hamid Osmany e 26B de Evgeny Romanov.

As entrevistas eram semiestruturadas, apresentando um roteiro de perguntas previamente formulado (Material Suplementar), servindo como apoio para a criação de um diálogo entre o pesquisador e o entrevistado, onde este pudesse discorrer livremente sobre o assunto (Bonie e Quaresma 2005). As perguntas versaram sobre o perfil dos pescadores participantes, a periodicidade e os locais da pesca de $C$. taurus, o conhecimento específico sobre a reprodução e alimentação desses animais, e as percepções ecológicas dos pescadores frente a possíveis alterações na abundância das populações da espécie e, possíveis consequências ambientais.

No intuito de verificar as preferências alimentares dos tubarões-mangona, os entrevistados foram questionados sobre quais itens esses animais costumam se alimentar e se existiam interações desses animais com outras espécies. Já para a reprodução, os pescadores discorreram sobre os locais e época onde fêmeas grávidas eram capturadas.

Para identificar se a pesca do tubarão-mangona era feita de forma intencional, os pescadores mencionaram se saiam para pescar esses animais ou se pescavam aleatoriamente acompanhados de outra fauna de interesse, ou seja, se eram by-catch. 
Neste sentido, os pescadores relatavam os lugares onde realizavam os procedimentos de captura e a época favorável para pesca da espécie.

As percepções ecológicas dos pescadores sobre as alterações temporais na abundância populacional desta espécie foram avaliadas ao perguntar se acreditam que os estoques populacionais dessa espécie estão se esgotando ao longo do tempo. Para isso, foram solicitados que utilizassem seus conhecimentos ecológicos locais de forma cronológica, a fim de comparação com os tempos passados e os dias atuais, além de informarem qualitativamente como percebem os efeitos causados pela ausência desses animais no meio ambiente.

A descrição das capturas de $C$. taurus foi realizada através das quantidades de animais capturados por pescador para um evento de pesca; pelo peso dos animais em quilos e pelo comprimento dos animais em metros.

Análise dos dados- Com base nas variáveis captura, peso e comprimento dentro de um intervalo numérico, foram realizadas as médias das variáveis para cada pescador, e posteriormente foi calculada a média e desvio padrão de cada variável para todos os entrevistados. A análise quantitativa foi realizada a partir das médias e percentuais afim de uniformização das respostas (Silva e Braga 2018). Foram realizadas correlações de Spearman entre cada uma das três variáveis dependentes (capturas, peso e comprimento) com as variáveis explicativas "Idade do pescador" e “Tempo de experiência de pesca”. Essas correlações foram realizadas no Programa Statistica 4.0.

De forma qualitativa todas as informações coletadas através dos questionamentos foram consideradas, podendo assim obter um diagnóstico sobre os pontos de interesse da pesquisa, desta forma, seguindo uma tendência de união das diversas competências individuais.

Ética- O estudo foi aprovado pelo Comitê de Ética em Pesquisas com Seres Humanos (CEPSH) sob o no 52308116.9.0000.0121. Todos os entrevistados assinaram o Termo de Consentimento Livre e Esclarecido (TCLE, Material suplementar) previamente ao início das entrevistas.

\section{RESULTADOS}

Perfil dos participantes- Foram entrevistados 24 pescadores artesanais ao largo da Grande Florianópolis. Os pescadores eram todos do sexo masculino e com faixa etária de 36 a 83 anos, sendo que a média de idade ( \pm desvio padrão) foi de 60,4 $( \pm 13,6)$ anos e a experiência de pesca foi de $44,3( \pm 13,8)$ anos, tendo início nessa atividade com 16,2 $( \pm 8,4)$ anos. 
Todos os entrevistados afirmaram que conhecem, pescavam ou já pescaram o tubarão-mangona, indicando a foto em que constava a espécie.

Características da pesca de Carcharias taurus- A pesca intencional de C. taurus na Grande Florianópolis foi descrita por $75 \%$ dos pescadores, $25 \%$ não praticava a pesca intencional, porém capturavam a espécie como fauna acompanhante (bycatch) de outras espécies alvo. A média de captura foi de 10,0 $( \pm 12,1)$ animais a cada evento de pesca. O tamanho médio dos animais que eram capturados consistia em 2,36 ( \pm 1,02) m e 97,o $( \pm 55,4) \mathrm{Kg}$. Não existiram correlações significativas entre as variáveis explanatórias "idade do pescador" e "tempo de experiência" com as variáveis dependentes: quantidades de capturas, tamanho e comprimento desses animais (Tabela 1).

Tabela 1. Resultados das análises de Correlação de Spearman das variáveis: "idade do pescador" e "tempo de experiência de pesca" relacionadas com as variáveis: tamanho de captura por dia, comprimento dos animais capturados e peso dos animais capturados.

\begin{tabular}{lccc}
\hline Analises de Correlação & $\mathbf{R}^{2}$ & $\mathbf{r}$ & $\mathbf{P}$ \\
\hline Idade X Captura & 0,01 & 0,11 & 0,59 \\
Experiência X Captura & 0,00 & $-0,02$ & 0,89 \\
Idade X Peso & 0,07 & $-0,27$ & 0,18 \\
Experiência X Peso & 0,12 & $-0,35$ & 0,08 \\
Idade X Comprimento & 0,00 & 0,08 & 0,69 \\
Experiência X Comprimento & 0,00 & $-0,06$ & 0,77 \\
\hline
\end{tabular}

Os pescadores evidenciaram que $C$. taurus tinha uma temporada de pesca na região da Grande Florianópolis, sendo que $83 \%$ deles apontaram que ela ocorria entre novembro e fevereiro. Os pescadores relataram a ocorrência de pesca de tubarãomangona em dez diferentes localidades na região, sendo a mais frequente a Praia do Pântano do Sul (Figura 1). 


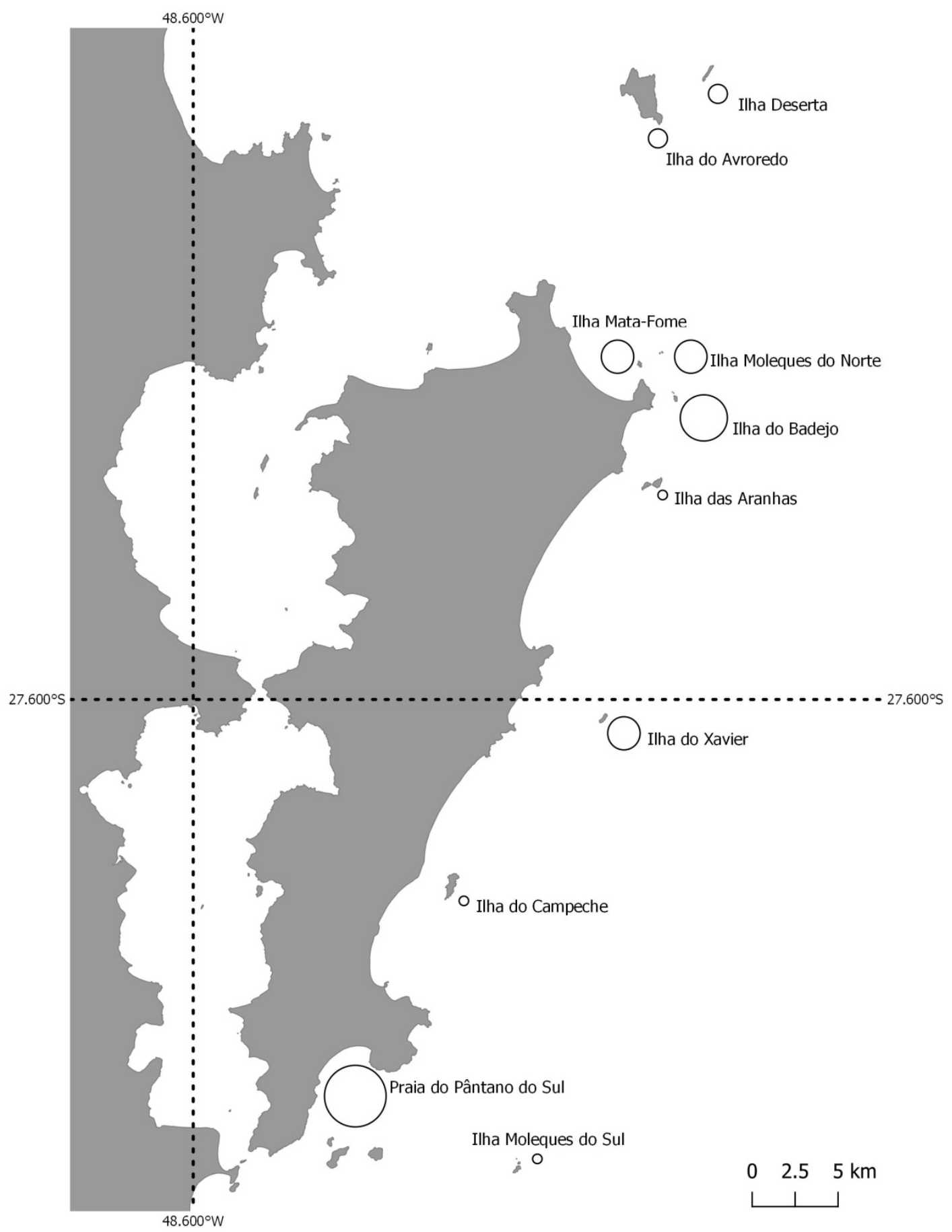

Figura 2. Mapa de caracterização dos locais de pesca de Carcharias taurus citados por pescadores entrevistados em sete comunidades pesqueiras da Grande Florianópolis. Bolhas variam em (mínimo: 1 citação; máximo: 8 citações).

Conhecimento ecológico tradicional- De acordo com $58 \%$ dos entrevistados, indivíduos de $C$. taurus faziam cerco de cardumes de peixes no momento da alimentação, aglomerando esses animais e aproximando os cardumes da costa. Porém, $42 \%$ não souberam informar se existe alguma interação desta espécie com outras espécies marinhas. Ao questionarmos sobre os itens alimentares do tubarão- 
mangona, o conhecimento dos entrevistados mostrou que este tubarão tem uma dieta generalista, mas majoritariamente constituída de diversas espécies de peixes ósseos, raias e lulas (Figura 2).

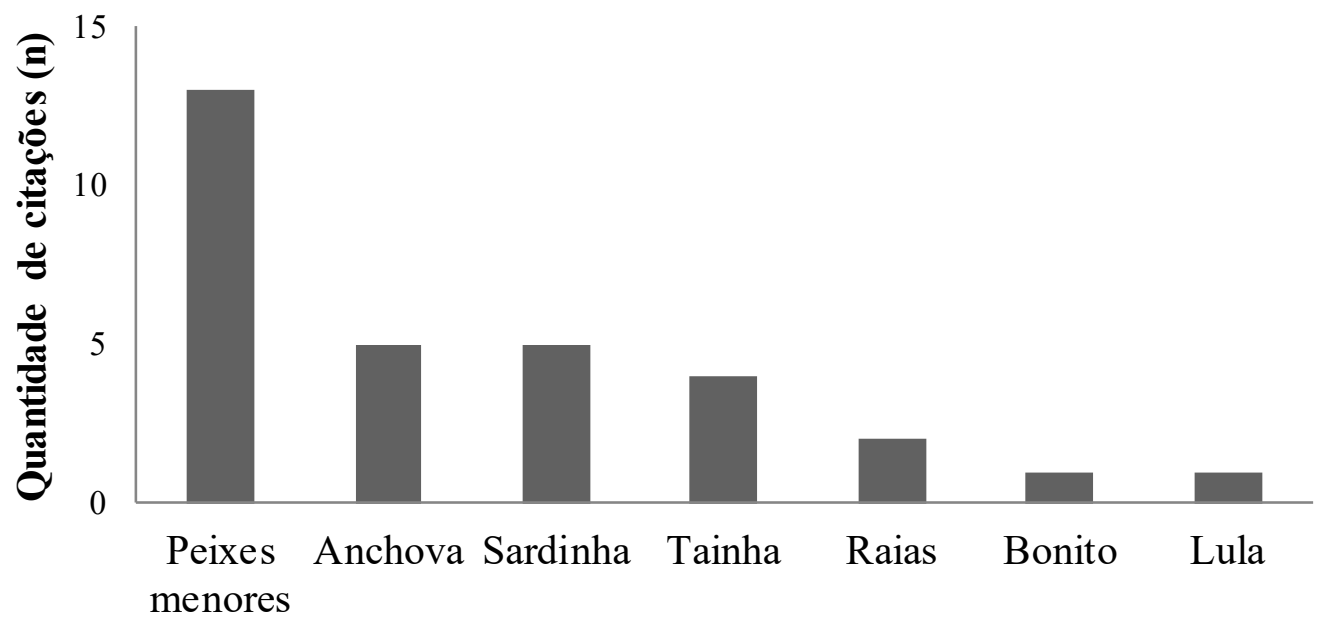

Figura 3. Descrição dos itens alimentares que compõe a dieta do tubarão-mangona de acordo com a percepção dos pescadores $(n=24)$.

Do total de pescadores, $46 \%$ afirmaram que o nascimento de filhotes acontecia entre os meses de novembro a fevereiro, outros $46 \%$ não souberam afirmar se a espécie se reproduzia na região e $8 \%$ acreditavam que acontecia em outros estados. Independentemente, $54 \%$ dos pescadores afirmaram que pescaram fêmeas grávidas. De acordo com os entrevistados, as fêmeas grávidas foram pescadas em média a $29,5( \pm 11,8)$ anos atrás sendo praticamente o mesmo tempo que as últimas capturas aconteceram, independentemente de serem fêmeas grávidas (9,0 $\pm 11,9$ anos). Todos os pescadores afirmaram que estes animais não são mais capturados atualmente.

Percepções ecológicas- A grande maioria dos pescadores (92\%) afirmou que acreditam que $C$. taurus está desaparecendo do ambiente marinho e (82\%) destes mencionaram as possíveis causas que levaram essa espécie a desaparecer do ambiente. Os pescadores foram capazes de reconhecer que a pesca em suas diferentes modalidades não sustentáveis como, a captura em números exorbitantes desses animais, a pesca de arrasto de fundo, a pesca na frota industrial e a pesca de nadadeiras, são os principais responsáveis pelo declínio da espécie na região. Somente $27 \%$ dos pescadores não quiseram mencionar o que teria levado a espécie a um declínio populacional.

Para os pescadores que observaram um desaparecimento desta espécie, $50 \%$ reconheceram que este desaparecimento acarreta algum tipo de efeito ao meio ambiente. Destes, 55\% afirmaram que o desaparecimento desta espécie pode gerar um desequilíbrio no meio ambiente, $18 \%$ dos pescadores mencionaram que 
na ausência desta espécie, ocorre o afastamento da costa dos peixes que são seus itens alimentares e $27 \%$ deles não entendem claramente quais efeitos poderiam ocorrer no meio ambiente. Dos outros 50\% dos entrevistados que observaram o desaparecimento da espécie, mas que não reconheceram que possa haver efeitos ambientais decorrentes do desaparecimento desta espécie, $45 \%$ deles disseram que, se há algum prejuízo, este se caracteriza por prejuízos financeiros ao pescador.

\section{DISCUSSÃO}

Este estudo mostrou que o tubarão-mangona foi amplamente capturado no passado ao largo da ilha de Santa Catarina, através da pesca artesanal, que é prática comum das populações tradicionais litorâneas. Os saberes tradicionais permitiram o reconhecimento de que esta espécie sofreu um declínio de suas populações na área de estudo ao longo dos anos. Também possibilitou uma análise sobre a conscientização dessas populações frente a este declínio, bem como, o entendimento sobre a importância ecológica da espécie para o meio ambiente. Da mesma forma, o conhecimento ecológico local, permitiu a caracterização da pesca desse tubarão no passado, e evidenciou aspectos sobre a alimentação e reprodução da espécie, reforçando prévios conhecimentos descritos na literatura e que podem contribuir com possíveis planos de manejo que visem a sua conservação.

A média de idade dos pescadores e a média de tempo de experiência com a atividade pesqueira artesanal foi muito próxima, evidenciando que a maioria dos pescadores aprendeu a pescar quando criança ou jovem. Isso sugere o largo conhecimento destes sobre a pesca e, que estes saberes são transmitidos de geração para geração, caracterizando o processo de aquisição empírica dos conhecimentos sobre a natureza (Diegues et al. 200o).

Há não correlação da idadee experiência dos pescadores com peso, comprimento e quantidade de captura podem ser explicados pelo fato da população ter diminuído abruptamente, ou seja, em uma janela temporal curta. Os entrevistados relataram que o tubarão-mangona era frequentemente capturado a cerca de 29 anos atrás, e os pescadores mais novos, provavelmente, ainda puderam ter tido contato com uma biomassa populacional parecida com a que os pescadores de mais idade presenciaram, visto que iniciavam a pesca com 16 anos em média. Desta maneira, eles podiam fazer capturas tão parecidas quanto os mais antigos faziam, sendo, portanto, antes que um efetivo declínio da espécie fosse observado (percebido de maneira diferente entre pescadores de diferentes idades). Por conseguinte, podemos inferir que em uma curta janela de tempo, esses animais, que eram largamente capturados, sofreram grande depleção de suas populações a ponto de, praticamente, não serem mais capturados hoje em dia. Os dados que apresentam a quantidade média de captura de $C$. taurus, mostram que a espécie foi amplamente pescada no passado, caracterizando que a pesca não sustentável para a espécie pode ter levado as populações deste tubarão a 
níveis críticos, uma vez que possui baixa capacidade de recuperação dos estoques a níveis considerados saudáveis (Lessa et al. 2005). A literatura aponta que esta espécie sofreu declínio em suas populações no Brasil a taxas de $80 \%$ em uma janela de três gerações, sendo que a redução da CPUE da espécie gira em torno de 90\% (ICMBioMMA 2018). Intensificando a problemática, os tamanhos médios dos animais que eram capturados indicam que se tratavam de adultos em idade reprodutiva, uma vez que fêmeas e machos maduros apresentam aproximadamente $220 \mathrm{~cm}$ (Lucifora et al. 2002) e a retirada desses pode ter comprometido a manutenção do potencial reprodutivo das populações.

O tubarão-mangona era capturado ao redor de nove ilhas ao largo de Florianópolis e na praia do Pântano do Sul, corroborando com outro estudo que aponta as ilhas da região como locais de pesca (Souza 1994). Isso também está de acordo com a expressividade amostral de dentes de $C$. taurus encontrados em restos faunísticos de um sítio arqueológico da região, evidenciando, além da abundância da espécie no passado, que o seu hábito costeiro o tornava de fácil captura para populações pré-coloniais desta região (Hayata et al. 2018).

Os pescadores citaram diversas espécies de peixes e outros grupos como itens alimentares de $C$. taurus. Esse conhecimento é corroborado com alguns estudos que caracterizam a espécie como generalista alimentar, pela presença de grande variedade de itens e por não apresentar predominância em um tipo alimentar específico (Compagno 1984; Gelsleichter et al. 1999; Lucifora 2009).

Os entrevistados relataram que esta espécie pratica cerco de cardumes dos peixes que são seus itens alimentares, promovendo, através dos seus hábitos de predação, a aproximação desses cardumes à costa, o que porventura faria com que esses animais se tornassem alvos fáceis para pesca. Afirmaram também, a ocorrência destes eventos como uma interação deste tubarão com outras espécies de peixes. $\mathrm{O}$ que se sabe é que existem agregações de indivíduos e que este comportamento pode estar associado tanto a hábitos alimentares quanto a reprodutivos (Barker et al. 2011). Nessas agregações de $C$. taurus ao redor do mundo, a reprodução está associada a ilhas, baías ou recifes costeiros (Alan 2002; Kneebone et al. 2018). Isso reforça que regiões costeiras de SC, com características físicas semelhantes, poderiam ser de grande importância para a reprodução da espécie, porém facilitava suas capturas. Os períodos reprodutivos da espécie, de acordo com os entrevistados, aconteciam entre os meses de novembro a fevereiro, e são semelhantes àqueles observados na literatura. Especialmente no mês de novembro, nas regiões sudeste e sul do Brasil foram feitas capturas de fêmeas grávidas e de neonatos, sugerindo que esta é uma possível época de reprodução (Lessa et al. 2005; ICMBio-MMA 2018). Além disso, evidências apontam a ocorrência de fêmeas grávidas nas águas mais quentes do sul do Brasil, onde acontecem os partos (Lucifora et al. 2002). A temporada de pesca do tubarão-mangona também foi descrita pelos entrevistados nesse mesmo período. Sabendo que a captura não sustentável desses animais representa riscos para manutenção dos estoques pesqueiros dessa espécie, essa época é prioridade para elaboração de planos de manejo, fiscalização e ações de conservação. 
Em vistas sobre o estado de conservação de C. taurus, os entrevistados mostraram reconhecer o declínio sofrido pela espécie ao longo dos anos. Sendo que grande parte destes, reconheceram possíveis causas que pudessem ter levado ao desaparecimento desta espécie. A pesca não sustentável em suas diferentes modalidades foi considerada a principal causa do declínio a partir do conhecimento dos pescadores. Ainda assim, apenas a metade destes pescadores reconheceu algum prejuízo ambiental decorrente do desaparecimento de $C$. taurus. Isto demonstra que apesar de reconhecerem o decréscimo das populações do tubarão-mangona e que existiu, até recentemente, uma pesca não sustentável na região do estudo, inclusive aquela que era praticada pelos próprios entrevistados, estes não reconhecem claramente os danos ambientais causados pela remoção dos predadores de topo. Consequentemente torna-se indispensável ações de educação ambiental que possam contornar essa deficiência. Ações nesse sentido podem possibilitar um maior conhecimento ecológico e sensibilização das populações tradicionais de pesca a cerca dos bens ambientais ao qual fazem uso. Não obstante irá trazer esclarecimentos sobre o estado atual de conservação das espécies sobrepescadas, e permitir o entendimento sobre a importância dos predadores de topo para a manutenção de um meio ambiente equilibrado e saudável. Ainda assim, em discordância com o padrão amostrado, houve relatos de entrevistados que não reconheceram os declínios da espécie na região. Isso mostra ou uma ausência de conhecimentos ou uma baixa intenção na conservação do grupo o que, independentemente, é uma problemática. Vale ressaltar que essa espécie consta na Portaria n ${ }^{\circ} 445$ (ICMBio 2014) e consequentemente não é permitida sua captura.

\section{AGRADECIMENTOS}

Agradecemos à Prof. - Dr. - Natalia Hanazaki pelas ajudas e discussões científicas e Myrna Faria Hornke, pelo apoio nas atividades de campo. Somos gratos à Mariana Beibe pela ajuda com o resumo em espanhol. Agradecemos também a todos os pescadores artesanais da Grande Florianópolis, que com suas histórias de vida e de profissão intimamente conectadas permitiram a realização deste estudo. Este estudo foi parte do trabalho de conclusão de curso da primeira autora.

\section{REFERENCIAS}

Alan F. 2002. Occurrence of Carcharias taurus in nursery areas of the Eastern and Western Cape, South Africa. Marine Freshwater Research 53:551-556.

Arruda R. 1999. "Populações tradicionais" e a proteção dos recursos naturais em Unidades de Conservação. Ambiente Sociedade, 2:79-93. 
Bannwart JP. 2014. A pesca artesanal marinha em Santa Catarina. Epagri, Boletim Didático 113:56.

Barbosa-Filho MLV, Schiavetti A, Alarcon DT, Costa-Neto EM. 2014. "Shark is the man!": ethnoknowledge of Brazil's South Bahia fishermen regarding shark behaviors. Journal Ethnobiology Ethnomedicine, 10:54.

Barker SM, Peddemors VM, Williamson JE. 2011. A video and photographic study of aggregation, swimming and respiratory behaviour changes in the Grey Nurse Shark (Carcharias taurus) in response to the presence of SCUBA divers. Marine and Freshwater Behaviour and Physiology 2:75-92.

Boni V, Quaresma SJ. 2005. Aprendendo a entrevistar: como fazer entrevistas em Ciências Sociais. EmTese, 2(1): 68-8o.

Branstetter S, Musick JA. 1994. Age and Growth Estimates for the Sand Tiger in the Northwestern Atlantic Ocean. Transactions of the American Fisheries Society, 123 (2): 242-254.

Bornatowski H, Braga RR, Barreto RP. 2018. Advances in marine vertebrate research in Latin America. Coastal Research Library, 22: 251-262.

Compagno LJ. 1984. Sharks of the world. An annotated and illustrated catalogue of shark species known to date. Part 2 - Carcharhiniformes. FAO Fisheries Synopsis 4: 251-655.

Costa L, Chaves P. 2006. Elasmobrânquios capturados pela pesca artesanal na costa sul do Paraná e norte de Santa Catarina, Brasil. Biota Neotropical, 6: 1-10.

Dent F, Clarke S. 2015. State of the global market for shark products. FAO Fisheries and Aquaculture Technical Papers. Rome, 590:196.

Diegues AC, Vieira RS, Silva V, Figols FA, Andrade D. 20oo. Biodiversidade e comunidades tradicionais no Brasil. MMA, COBIO, NUPAUB- USP, São Paulo 1: 211.

Drew JA. 2005. Use of traditional ecological knowledge in marine conservation. Conservation Biology 19:1286-1293.

Gelsleichter J, Musick JA, Nichols S. 1999. Food habits of the smooth dogfish, Mustelus canis, dusky shark, Carcharhinus obscurus, Atlantic sharpnose shark, Rhizoprionodon terraenovae, and the sand tiger, Carcharias taurus, from the northwest Atlantic Ocean. Environmental Biology of Fishes, 54:205-217. 
Goldman KJ, Branstetter S, Musick JA. 2006. A reexamination of the age and growth of sand tiger sharks, Carcharias taurus, in the western North Atlantic: the importance of ageing protocols and use of multiple back calculation techniques. Environmental Biology of Fishes, 77: 241-252.

Hayata MA, Mayer GB, Soares LE, d'Avila MG, Hornke MF, Freitas RHA. 2018. O grupo dos elasmobrânquios. In: InforMar - Tubarões e Raias, A importância dos elasmobrânquios para a qualidadedos ecossistemas marinhos a partir do conhecimento local. Martins TM, Tomazi AL, Brenuvida WW (Ed.). 1ed. Baía de Tijucas, Editora 3 de Maio, 8op.

IBAMA - Instituto Brasileiro do Meio Ambiente e dos Recursos Naturais Renováveis. 2011. Proposta de Plano de Gestão para o uso sustentável de Elasmobrânquios sobreexpotados ou ameaçados de sobre-explotação no Brasil. Brasília: 154.

ICMBio- Instituto Chico Mendes da Biodiversidade. 2014. Ministério do Meio Ambiente- Lista nacional oficial de espécies da fauna brasileira ameaçadas de extinção, Peixes e Invertebrados Aquáticos. Diário Oficial da União, Brasil:126-130.

ICMBio-MMA. Instituto Chico Mendes de Biodiversidade. 2018. Livro vermelho da fauna brasileira ameaçada de extinção. 1ed., Brasília: IV- Peixes, 1235p.

Johannes RE, Freeman MMR, Hamilton RJ. 2008. Ignore fishers' knowledge and miss the boat. Fish and Fisheries, 1:257-271.

Johnson M. 1992. Capturing traditional environmental knowledge, Copyright, Ottawa, 1:94.

Kneebone J, Winton M, Danylchuk A, Chisholm J, Skomal GB. 2018. An assessment of juvenile sand tiger (Carcharias taurus) activity patterns in a seasonal nursery using accelerometer transmitters. Environmental Biology of Fishes, 101(12):1739-1756.

Lack M, Sant G. 2006. World Shark Catch, Production and Trade 1990-2003. Paper to the Technical Workshop on the Conservation and Management of Sharks, Intersessional Shark Working Group of the CITES Animals Committee. Australian Department of the Environment and Heritage and Traffic. Oceania:29.

Lessa R, Vooren CM, Araújo MLG, Kotas JE, Almeida PC, Filho GR, Santana FM, Gadig OB, Sampaio C, Almeida Z, Almeida M, Rosa RS. 2005. Plano nacional de ação para a conservação e o manejo dos estoques de peixes elasmobrânquios no Brasil. Sociedade Brasileira para o Estudo de elasmobrânquios - SBEEL, 1:10o 
Lucifora L, Menni RC, Escalante H. 2002. Reproductive ecology and abundance of the sand tiger shark, Carcharias taurus, from the southwestern Atlantic. ICES Journal of Marine Science, 59:553-561.

Lucifora LO, García VB, Escalante AH. 2009. How can feeding habits of the sand tiger shark influence the success of conservations programs?. Animal Conservation. 12: 291 $-301$.

Pereira B, Diegues A. 2010. Conhecimento de populações tradicionais como possibilidade de conservação da natureza: uma reflexão sobre a perspectiva da etnoconservação. Desenvolvimento e Meio Ambiente, 22:37-50.

Pollard D, Smith A. 2009. Carchariastaurus. The IUCN Red List of Threatened Species 2009: Disponível em <http://dx.doi.org/10.2305/IUCN.UK.2009-2.RLTS. T3854A10132481.en> Acesso em 31 de outubro de 2018.

Reis-Filho JA, Freitas RHA, Loiola M, Leite L, Soeiro G, Oliveira HHQ, Sampaio CLS, Nunes JACC, Leduc AOHC. 2016. Traditional fisher perceptions on the regional disappearance of the largetooth sawfish Pristis pristis from the central coast of Brazil. Endanger Species Research, 29:189-200.

Silva JT, Braga TMP. 2018. Etnoictiologia de Pescadores Artesanais da Comunidade Surucuá (Reserva Extrativista Tapajós-Arapiuns). Amazônia - Revista Antropológica, 9:238-257.

Souza HS. 1994. O homem da ilha e os pioneiros da caça submarina, 1ed., Florianópolis: Editora Dehon, 322p. 